PROCEEDINGS OF THE

AMERICAN MATHEMATICAL SOCIETY

Volume 127, Number 2, February 1999, Pages 343-348

S 0002-9939(99)04873-X

\title{
PRESENTATIONS FOR SUBGROUPS OF ARTIN GROUPS
}

\author{
WARREN DICKS AND IAN J. LEARY \\ (Communicated by Ronald M. Solomon)
}

\begin{abstract}
Recently, M. Bestvina and N. Brady have exhibited groups that are of type FP but not finitely presented. We give explicit presentations for groups of the type considered by Bestvina-Brady. This leads to algebraic proofs of some of their results.
\end{abstract}

Let $\Delta$ be a finite flag complex, that is, a finite simplicial complex that contains a simplex bounding every complete subgraph of its 1-skeleton. The associated rightangled Artin group $G_{\Delta}$ is the group given by the presentation with generators the vertex set of $\Delta$, and relators the commutators $[v, w]$ for each pair of adjacent vertices in $\Delta$. For example, the $n$-simplex corresponds to a free abelian group of rank $n+1$, a complex consisting of $n$ points corresponds to the free group $F_{n}$ of rank $n$, the group corresponding to the square is $\left(F_{2}\right)^{2}$, and the group corresponding to the octahedron is $\left(F_{2}\right)^{3}$.

Provided that $\Delta$ is non-empty, there is a homomorphism from $G_{\Delta}$ onto the integers, that takes every generator to 1 . The group $H_{\Delta}$ is defined to be the kernel of this homomorphism. Remarkable recent work of Mladen Bestvina and Noel Brady has shown that the homological finiteness properties of the group $H_{\Delta}$ are controlled by the topology of the complex $\Delta$ [1]. They show that $H_{\Delta}$ is finitely generated if and only if $\Delta$ is connected, $H_{\Delta}$ is finitely presented if and only if $\Delta$ is 1-connected, and $H_{\Delta}$ is of type $F P(n)$ if and only if $\Delta$ is $(n-1)$-acyclic. Precursors of this result include J. Stallings' group that is finitely presented but not of type $F P(3)$, which is $H_{\Delta}$ in the case when $\Delta$ is the octahedron [8], and R. Bieri's group of type $F P(n)$ but not of type $F P(n+1)$, which is $H_{\Delta}$ in the case when $\Delta$ is a join of $(n+1)$ pairs of points (section 2.6 of [2]).

The arguments used by Bestvina and Brady are geometric, and they do not give presentations for the groups that they consider. Theorem 1 of this paper gives a presentation for $H_{\Delta}$ for any connected $\Delta$. The generators in the presentation are the edges of $\Delta$, and each 1-cycle in $\Delta$ gives rise to an infinite family of relators. In the case when $\Delta$ is simply connected, it is shown how to reduce this presentation to a finite one. This gives an independent and purely algebraic proof that $H_{\Delta}$ is finitely presented when $\Delta$ is simply connected. It would be interesting to give a similar proof of the converse. In Proposition 4 and Corollary 5, we review some

Received by the editors May 17, 1997.

1991 Mathematics Subject Classification. Primary 20F36; Secondary 20E07, 20 F32.

Key words and phrases. Artin group, presentation.

W. Dicks acknowledges support from the DGICYT (Spain) through grant number PB93-0900.

I. Leary acknowledges support from the Nuffield Foundation through grant number SCI/180/96/127, and from EPSRC grant number GR/L06928. 
results concerning the homology of $G_{\Delta}$. Using an argument due to Stallings, we deduce that when the Euler characteristic of $\Delta$ is not equal to that of a point, the rational cohomology of $H_{\Delta}$ cannot be finite dimensional.

Definition. For $e$ a directed edge of $\Delta$, let $\iota e$ (resp. $\tau e$ ) denote the initial (resp. terminal) vertex of $e$.

Theorem 1. Let $\Delta$ be connected. The group $H_{\Delta}$ has a presentation with generators the set of directed edges of $\Delta$, and relators all words of the form $e_{1}^{n} e_{2}^{n} \ldots e_{l}^{n}$, where $l, n \in \mathbb{Z}, n \neq 0, l \geq 2$, and $\left(e_{1}, \ldots, e_{l}\right)$ is a directed cycle in $\Delta$. In terms of the given generators for $G_{\Delta}, e=\iota e(\tau e)^{-1}$.

Proof. Let $H_{\Delta}^{\prime}$ be the group presented as in the statement. Cycles of length two in $\Delta$ have the form $(e, \bar{e})$, where $\bar{e}$ is the same edge as $e$ with its opposite orientation. The relation $e \bar{e}=1$ implies that for each edge $e, \bar{e}=e^{-1}$ in $H_{\Delta}^{\prime}$. These relations could of course be used to halve the size of the generating set at the expense of adding some signs in the relators. We shall apply these relations as necessary without comment in the sequel. From the symmetrical form of the relators in $H_{\Delta}^{\prime}$, it follows that there is an endomorphism $\xi$ of $H_{\Delta}^{\prime}$ which sends each directed edge $e$ to $e^{-1}$. Moreover, $\xi^{2}=1$, and so $\xi$ is an automorphism.

Define a homomorphism $\phi$ from $H_{\Delta}^{\prime}$ to $G_{\Delta}$ by $\phi(e)=\iota e(\tau e)^{-1}$. This does define a homomorphism, since if $\left(e_{1}, \ldots, e_{l}\right)$ is a directed cycle, with $\iota e=a_{i}$ and $\tau e=a_{i+1}$, then

$$
\phi\left(e_{1}^{n} \cdots e_{l}^{n}\right)=\left(a_{1}^{n} a_{2}^{-n}\right)\left(a_{2}^{n} a_{3}^{-n}\right) \cdots\left(a_{l}^{n} a_{1}^{-n}\right)=1 .
$$

The image of $\phi$ is contained in $H_{\Delta}$. Conversely, any element $w$ of $H_{\Delta}$ is expressible in the form $w=a_{1}^{n(1)} a_{2}^{n(2)} \cdots a_{m}^{n(m)}$, where $n(1)+\cdots+n(m)=0$. If $\left(e_{1}, \ldots, e_{r}\right)$ is a directed path from $a_{m}$ to $a_{m-1}$, then $\phi\left(e_{1}^{-n(m)} \cdots e_{r}^{-n(m)}\right)=a_{m}^{-n(m)} a_{m-1}^{n(m)}$. By induction on $m$ it follows that any element of $H_{\Delta}$ is in the image of $\phi$.

It remains to prove that $\phi$ is injective. To do this, our strategy is as follows. Define an extension $G_{\Delta}^{\prime}$ with kernel $H_{\Delta}^{\prime}$ and quotient $\mathbb{Z}$, extend $\phi$ to a homomorphism $\tilde{\phi}$ from $G_{\Delta}^{\prime}$ to $G_{\Delta}$, and finally show that $\tilde{\phi}$ is an isomorphism by exhibiting an inverse.

For vertices $a$ and $b$ of $\Delta$, define $p(a, b)$ to be an element of $H_{\Delta}^{\prime}$ represented by the word $e_{1} \cdots e_{n}$, where $\left(e_{1}, \ldots, e_{n}\right)$ is a choice of directed edge path from $a$ to $b$. The hypothesis that $\Delta$ be connected ensures that there is such a choice. Note that different choices of directed edge path give rise to the same element of $H_{\Delta}^{\prime}$. Fix a vertex $a$, and define an endomorphism $\psi_{a}$ of $H_{\Delta}^{\prime}$ by $\psi_{a}(e)=p(a, \iota e) e p(\iota e, a)$. It must be shown that this does define an endomorphism. First note that

$$
\psi_{a}\left(e^{n}\right)=p(a, \iota e) e^{n} p(\iota e, a)=p(a, \iota e) e^{n+1} p(\tau e, a) .
$$

Now if $\left(e_{1}, \ldots, e_{l}\right)$ is any directed path from $b$ to $b^{\prime}$, then for any non-zero integer $n$,

$$
\psi_{a}\left(e_{1}^{n} e_{2}^{n} \cdots e_{l}^{n}\right)=p\left(a, \iota e_{1}\right) e_{1}^{n+1} p\left(\tau e_{1}, a\right) \cdots p\left(a, \iota e_{l}\right) e_{l}^{n+1} p\left(\tau e_{l}, a\right),
$$

which telescopes to the equation

$$
\psi_{a}\left(e_{1}^{n} \cdots e_{l}^{n}\right)=p(a, b) e_{1}^{n+1} e_{2}^{n+1} \cdots e_{l}^{n+1} p\left(b^{\prime}, a\right) .
$$

By considering the case when $b=b^{\prime}$, it may be seen that $\psi_{a}$ defines an endomorphism of $H_{\Delta}^{\prime}$. 
To show that $\psi_{a}$ is an automorphism, we compute its composite with the automorphism $\xi$ defined by $\xi(e)=e^{-1}$. The case $n=-1$ of the equation above gives

$$
\psi_{a} \xi\left(p\left(b, b^{\prime}\right)\right)=p(a, b) p\left(b^{\prime}, a\right) .
$$

Hence for any edge $e$,

$$
\begin{aligned}
\psi_{a} \xi \psi_{a} \xi(e) & =\psi_{a} \xi(p(a, \iota e) p(\tau e, a)) \\
& =\psi_{a} \xi(p(a, \iota e)) \psi_{a} \xi(p(\tau e, a)) \\
& =(p(a, a) p(\iota e, a))(p(a, \tau e) p(a, a)) \\
& =e
\end{aligned}
$$

Thus $\psi_{a} \xi$ has order two, so is an automorphism, and it follows that $\psi_{a}$ is an automorphism.

Let $G_{\Delta}^{\prime}$ be the extension with kernel $H_{\Delta}^{\prime}$ and infinite cyclic quotient, generated by $a^{\prime}$, where the conjugation action of $a^{\prime}$ is given by $a^{\prime} h a^{\prime-1}=\psi_{a}(h)$. For every directed edge $e, \phi\left(\psi_{a}(e)\right)=a \phi(e) a^{-1}$, and so $\phi$ may be extended to a surjective homomorphism $\tilde{\phi}: G_{\Delta}^{\prime} \rightarrow G_{\Delta}$ by setting $\tilde{\phi}\left(a^{\prime}\right)=a$. Define a map $\theta: G_{\Delta} \rightarrow G_{\Delta}^{\prime}$ on the generators by $\theta(b)=p(b, a) a^{\prime}$. For any directed edge $e, p(\iota e, a)=e p(\tau e, a)$ in $H_{\Delta}^{\prime}$, and hence $\theta(\iota e)=e \theta(\tau e)$. It is also true that

$$
\begin{aligned}
\theta(\tau e) e & =p(\tau e, a) a^{\prime} e \\
& =p(\tau e, a) \psi_{a}(e) a^{\prime} \\
& =p(\tau e, a) p(a, \iota e) \operatorname{ep}(\iota e, a) a^{\prime} \\
& =p(\iota e, a) a^{\prime}=\theta(\iota e) .
\end{aligned}
$$

Hence for each directed edge $e, \theta(\tau e)^{-1} \theta(\iota e)=e=\theta(\iota e) \theta(\tau e)^{-1}$. It follows that $\theta$ extends to a homomorphism from $G_{\Delta}$ to $G_{\Delta}^{\prime}$. Finally, it may be shown that $\theta$ is an inverse to $\tilde{\phi}$, which is therefore an isomorphism.

Proposition 2. For a directed cycle $c=\left(e_{1}, \ldots, e_{l}\right)$ of length $l$ in $\Delta$, write $c^{[n]}$ for the relator $e_{1}^{n} \cdots e_{l}^{n}$ in the above presentation for $H_{\Delta}$. The presentation may be simplified as follows.

(a) When $l=2$, the relators $c^{[n]}$ are consequences of $c^{[1]}$.

(b) When $l=3$, the relators $c^{[n]}$ are consequences of the relators $c^{[1]}$ and $c^{[-1]}$.

(c) Let $c_{1}, \ldots, c_{m}$ be directed cycles such that the normal subgroup of $\pi_{1}(\Delta)$ generated by the conjugacy classes of $c_{1}, \ldots, c_{m}$ is the whole of $\pi_{1}(\Delta)$. Then each relator $c^{[n]}$ is a consequence of the (finitely many) relators given in (a) and (b), together with the relators $c_{i}^{[n]}$ for all $n$ and $1 \leq i \leq m$.

Proof. As remarked earlier, the relation $e \bar{e}=1$ implies that $\bar{e}=e^{-1}$. The relations $e^{n} \bar{e}^{n}$ for all $n$ are consequences of this. Now suppose that $(e, f, g)$ is a directed triangle in $\Delta$. The relation efg $=1$ implies that $g=f^{-1} e^{-1}$. Substituting for $g$, the relator $e^{-1} f^{-1} g^{-1}$ becomes the commutator $e^{-1} f^{-1} e f$. It follows that the group generated by $e, f$ and $g$ subject to just these two relations is isomorphic to $\mathbb{Z} \times \mathbb{Z}$, via an isomorphism sending $e, f, g$ to $(1,0),(0,1)$ and $(-1,-1)$. For each $n$, the relation $e^{n} f^{n} g^{n}=1$ already holds in this group.

If the cycles $c$ and $c^{\prime}$ differ only in their choice of starting point, i.e., $c=$ $\left(e_{1}, \ldots, e_{l}\right)$ and $c^{\prime}=\left(e_{r+1}, \ldots, e_{l}, e_{1}, \ldots, e_{r}\right)$, then the relator $c^{\prime[n]}$ is a conjugate of the relator $c^{[n]}$. Similarly, if $c^{\prime}$ is equal to $c$ but in the opposite orientation, then 
$c^{\prime[n]}$ may be obtained from the inverse of the relator $c^{[n]}$ by applying the relators listed in (a). If $c$ is a composite cycle, $c=c^{\prime} \cdot c^{\prime \prime}$, then $c^{[n]}$ is equal to $c^{[n]} c^{\prime \prime[n]}$. Let $p$ be any directed edge path in $\Delta$, and let $\bar{p}$ be the opposite path. By induction on the length of the path $p$, the relators $(\bar{p} p)^{[n]}$ are consequences of the finitely many relators listed in (a).

For convenience, choose a basepoint $a$ for $\Delta$. For each path $p$ from $a$ to the initial point of $c_{i}$, the relator $\left(p c_{i} \bar{p}\right)^{[n]}$ is a consequence of $c_{i}^{[n]}$ and the relators listed in (a). By assumption, composites of based cycles of this form and their inverses suffice to construct an element of every based homotopy class of cycles in $\Delta$. By the remarks in the previous paragraph any such relator is deducible from the relators given in (a)-(c). To complete the proof, it suffices to show that whenever $c$ and $c^{\prime}$ are homotopic cycles, the relator $c^{[n]}$ is a consequence of $c^{[n]}$ and the relators listed in (a) and (b). View a simplicial homotopy from $c$ to $c^{\prime}$ as a triangulation of $S^{1} \times I$ together with a labelling of its directed edges by generators of $H_{\Delta}$ and the identity element, having the following properties: the labelling of $S^{1} \times\{0\}$ spells $c$; the labelling of $S^{1} \times\{1\}$ spells $c^{\prime}$; the labelling of each triangle is of one of the following forms: $(e, f, g)$ for some triangle $(e, f, g)$ in $\Delta$, or $(e, \bar{e}, 1)$, or $(1,1,1)$. Now move from $S^{1} \times\{0\}$ to $S^{1} \times\{1\}$ 'one simplex at a time', at each stage either removing a 1 -simplex that is connected to the remaining complex only at one end, or removing a 2-simplex that has an edge in the boundary of the remaining complex, together with that edge. This gives rise to a sequence $c=d_{0}, d_{1}, \ldots, d_{k}=c^{\prime}$ of cycles in $\Delta$ such that $d_{i+1}$ differs from $d_{i}$ in only one of the following ways:

$$
\begin{aligned}
& d_{i+1}=d_{i}, \\
& d_{i+1}=d^{\prime} \cdot e^{-e} \cdot d^{\prime \prime} \quad \text { and } d_{i}=d^{\prime} \cdot d^{\prime \prime}, \quad \text { for some edge } e \text {, } \\
& d_{i}=d^{\prime} . e . \bar{e} \cdot d^{\prime \prime} \quad \text { and } \quad d_{i+1}=d^{\prime} \cdot d^{\prime \prime}, \quad \text { for some edge } e, \\
& d_{i+1}=d^{\prime} \cdot \bar{g} \cdot \bar{f} \cdot d^{\prime \prime} \quad \text { and } d_{i}=d^{\prime} \cdot e \cdot d^{\prime \prime}, \quad \text { for some triangle }(e, f, g) \text {. }
\end{aligned}
$$

In each case it may be seen that the relator $d_{i+1}^{[n]}$ is a consequence of $d_{i}^{[n]}$ together with the relators of types (a) and (b).

Corollary 3. When $\Delta$ is simply connected, $H_{\Delta}$ is finitely presented. There is a presentation for $H_{\Delta}$ with generators the directed edges of $\Delta$, and relators

$$
\begin{array}{lll}
e \bar{e} & \text { for every edge e, } \\
\text { efg }, \quad g f e & \text { for every directed triangle }(e, f, g) .
\end{array}
$$

Proof. This is a special case of Proposition 2(c).

Remark. Let $K_{\Delta}$ be the group generated by the directed edges of the complex $\Delta$ subject only to the relations of types (a) and (b) of Proposition 2. There is a natural map from $K_{\Delta}$ onto $H_{\Delta}$, which is an isomorphism when $\Delta$ is simply connected. To show that $H_{\Delta}$ cannot be finitely presented when $\Delta$ is not simply connected, it would suffice to show that $\operatorname{ker}\left(K_{\Delta} \rightarrow H_{\Delta}\right)$ is not finitely generated as a normal subgroup of $H_{\Delta}$. It would be very interesting if one could do this algebraically. The group $K_{\Delta}$ arises implicitly in the work of Mladen Bestvina and Noel Brady, as the fundamental group of a 'level set' [1]. Jim Howie constructs another 2-complex with fundamental group $K_{\Delta}$, and uses this to give an alternative proof that $H_{\Delta}$ is $F P(2)$ when $\Delta$ is 1 -acyclic, in $[6]$.

Definition. For a set $V$, let $\Lambda^{*}(V)$ be the exterior algebra generated (in degree one) by the functions from $V$ to $\mathbb{Z}$. For a simplicial complex $\Delta$, the exterior face 
$\operatorname{ring} \Lambda_{\Delta}^{*}$ is the quotient $\Lambda(V) / I$, where $V$ is the vertex set of $\Delta$, and $I$ is the ideal generated by all monomials of the form $v_{0} \cdots v_{n}$ such that $\left(v_{0}, \ldots, v_{n}\right)$ is not an $n$-simplex of $\Delta$.

The exterior face ring $\Lambda_{\Delta}^{*}$ is a contravariant functor of $\Delta$, and is graded, since $I$ is generated by homogeneous elements. For each $i>0, \Lambda_{\Delta}^{i}$ is a free abelian group of rank equal to the number of $(i-1)$-simplices in $\Delta$. The complex $\Delta$ is a flag complex if and only if $I$ is generated by monomials of degree two.

Proposition 4 (Kim-Roush). The integral cohomology ring of $G_{\Delta}$ is naturally isomorphic to the exterior face ring of $\Delta$.

Proof. Since this proposition has already appeared in [7], p. 185, and in [4], pp. 227228 , we merely sketch the proof. When $\Delta$ is a simplex, $G_{\Delta}$ is free abelian and $H^{*}\left(G_{\Delta}\right)=\Lambda(\operatorname{Hom}(G, \mathbb{Z}))=\Lambda_{\Delta}$. When $\Delta$ is not a simplex, $\Delta=\Delta_{1} \cup \Delta_{2}$ for smaller flag complexes $\Delta_{1}$ and $\Delta_{2}$, with $\Delta_{3}=\Delta_{1} \cap \Delta_{2}$. This gives rise to a decomposition of $G$ as a free product with amalgamation $G=G_{1} *_{G_{3}} G_{2}$, where $G_{i}=G_{\Delta_{i}}$. The claim for $\Delta$ follows by a Mayer-Vietoris argument. $[3]$.

The following result is due to C. Droms [5]; see also I. Chiswell's generalization

Corollary 5 (Droms). The Euler characteristic of $G_{\Delta}$ is equal to $1-\chi(\Delta)$.

Corollary 6. If the rational cohomology of $H_{\Delta}$ is finite-dimensional, then $\chi(\Delta)=$ 1.

Proof. Let $G=G_{\Delta}$ and $H=H_{\Delta}$. The Mayer-Vietoris sequence for $G$ expressed as an HNN-extension with base group $H$ is a long exact sequence:

$$
\cdots \rightarrow H^{i-1}(H) \rightarrow H^{i-1}(H) \rightarrow H^{i}(G) \rightarrow H^{i}(H) \rightarrow H^{i}(H) \rightarrow \cdots .
$$

In the case when $H^{*}(H)=H^{*}(H, \mathbb{Q})$ is finite-dimensional, it follows that the Euler characteristic of $G$ must be zero, and hence that $\chi(\Delta)=1$.

Corollary 7 (Bestvina-Brady). If $\Delta$ is both connected and simply connected, and $\chi(\Delta) \neq 1$, then $H_{\Delta}$ is finitely presented but not of type FP.

\section{ACKNOWLEDGEMENT}

The authors thank the referee for carefully reading an earlier version of this paper.

\section{REFERENCES}

[1] M. Bestvina and N. Brady, Morse theory and finiteness properties of groups, to appear in Invent. Math.

[2] R. Bieri, Homological dimension of discrete groups, Queen Mary College Mathematics Notes, University of London (1976). MR 57:6224

[3] I. M. Chiswell, The Euler characteristic of graph products and of Coxeter groups, Discrete groups and Geometry (Birmingham 1991), London Math. Soc. Lecture Notes 173, 36-46, Cambridge Univ. Press, Cambridge (1992). MR 94a:05090

[4] W. Dicks, An exact sequence for rings of polynomials in partly commuting indeterminates, J. Pure Appl. Algebra 22, 215-228 (1981). MR 82m:16014

[5] C. Droms, Subgroups of graph groups, J. Algebra 110, 519-522 (1987). MR 88m:05046

[6] J. Howie, Bestvina-Brady groups and the plus construction, preprint (1997). 
[7] K. H. Kim and F. W. Roush, Homology of certain algebras defined by graphs, J. Pure Appl. Algebra 17, 179-186 (1980). MR 82e:05114b

[8] J. R. Stallings, A finitely presented group whose 3-dimensional integral homology is not finitely generated, Amer. J. Math. 85, 541-543 (1963). MR 28:2139

Departament de Matemàtiques, Universitat Autònoma de Barcelona, E-08193 Bellaterra (BArCElona), Spain

E-mail address: dicks@manwe.mat.uab.es

Faculty of Mathematical Studies, University of Southampton, Southampton, SO17 1BJ, United Kingdom

E-mail address: ijl@maths.soton.ac.uk 